ter löslichen Salze bald ganz ausgewaschen wurden. Bei der uicht erhitzten Verbindung hiugegen geschielit dic Zersetzung hei weitem langsamer, und geschieht uberhaupt nur dadurch endlich vollständig, dafs die Lösung des zersetzten Theils durch neucs Wasser ersetzt wird.

Wird daher das Pulver des nicht erhitzten Polybalits mit wenigem kalten Wasser ubergossen, wodurch nur ein kleiner Theil desselben zersetzt wird, und läfst man das Ganze stehen, so bleibt der grö[ste, der nicht zersetzte Theil des Salzes, auch noch nach sehr langer Zeit unzersetzt, weil die nicht erhitzte Verbindung in der Lösungdes zersetzten Theils unlüslich ist, was beim erhitzten, und dadurch seines Krystallwassers beraubten Polyhalit nicht der Fall ist.

Es glückte mir nicht, den Polyhalit künstlich darzustellen. Bei den Versuchen, ihn aus seinen Bestandtheilen zu erzeugen, wurde, wie ich weiter unten zeigen werde, ein anderes Salz erbalten.

(Fortsetzang fulgt.)

II. Ueber die Krystallform einiger salpetersauren und kohlensnuren Salze; ron Frankenheirn.

Di ie Methode der Normalen in der Krystallographie steht zwar dem gewöhulichen Verfähren, bei welchem die Punkte bestinmt werden, wo die Axen von den Flächen bestiment werden, an Anschaulichkeit nach, ist ihm aber zur Berechnung der Zouen und Winkel, sowic zu jedem wissenschaftlichen Zwecke weit vorzuzieben. Ich werde mich daher in den folgenden AuIsätzeu auch bei der Bezeichnung der Grundformen und der Flächen immer der Normalen bedienen, um so mehr als dieses ron mir schon in meiner 1838 erschienenen Cohäsions - Lehre und noch ältcren $\mathrm{Ab}$ - 
handlungen angewendete Verfaliren seitlem von mehreren Krystallographen, unter Anderen von Miller, fast ohne Abainderung angenoumen ist.

Beide Methoden, nämlich die der Normalen, wo die Lage der auf den Flächen perpendikular stehenden I.inien durch gewoubnliche dreien Axen parallele Coordiraten bestimnt wird, und die ron G. Rose modificirte Bezcichnungs - Weise ron Weils, kömnen ührigeus leicht auf eitıander reducirt werden, indem eine Fläche, deren Bezcirh. nung $a a: \beta b: \gamma c$ ist, einer Normale entspricht, deren (oordinaten $d A: e B: f c$ sind, wenn $d=\frac{1}{a} ; e=\frac{1}{\beta} ; f=\frac{1}{;}$.

Auch die Grund-Verhaltnisse $a: b: c$ und $A: B: C$ stehen in einer sehr einfachen Bezichung.

Ich setze $A$ immer $=1$ und nehme dafür in den tetragonalen und hexagonalen Systemen die Hauptaxen, in dem monoklinisclien Systeme die ebenfalls durch die Symmetrie ihrer Lage ausgezeichnete Linie, welche allen Prismen parallel, auf alle schiefen Endflichen perpendikular steht. In dem isoklinischen (ein- und einaxigen) und triklinischen Systeme ist $A$ die kleinste, $C$ die gröfste der drei Axen.

Da es bei der Bezcichnung der einzchnen Normalen übertlüssig wärc, die Zeichen $A, B, C$ zu wiederholen, so wird von ciner Fläche, welcho bei Weifs die Bezeichnung $[6 a: 3 b: 2 c]$ oder, wie er gewöhnlich setzt, $\left[a: \frac{1}{2} b: \div c\right]$ haben würde, die Normale das Zeichen [ $\left[\begin{array}{lll}1 & 2 & 3\end{array}\right]$ haben. Auch dic Parenthese ist in der Regel überflüssig. Da ein Coëflicient, der zwei Ziffern hätte, schr selten oder gar nicht vorkommt, so ist ein Tremungszeichen zwischen ihuen ebenfalls unnothig.

Nicht selten ist der Krystall in der Richtung der klein. sten der drei Axen am stärksten ausgedehnt; diese erscheint alsdann als die Hauptrichtung und wird gewöhnlich als dic Hauptaxe des Krystalls angesehen und vertical gestellt. Diese Stellung, welcher die Mineralogen eine, wie mir scheint, viel zu hohe Wichtigkeit beilegen, hat für unsere Aufgabe natürlich gar keine Bedeutung. Wichtiger ist cs, 
dafs dic Projectionen der optischen Elasticitäts - Axen der Hauptrichtung der Prismen gewöhnlich perpendikular und parallel sind.

Der gewöhuliche Salpeter hat auf rechtwinklige Axen bezogen das Grund - Verhältnifs

$A: B: C=1: 1,402: 2,397$ nach Miller.

Statt dieser Zahlen ist es bequener die Logarithmen von $B, C$ und $C: B$ zu setzen, also für

$\begin{array}{lrrrr}\text { Salpeter } & 0,1468 & \mathbf{0 , 3 7 9 6} & \mathbf{0 , 2 3 2 8 ,} \text { Miller, } \\ \text { Arragonit } & 1587 & \mathbf{3 6 5 3} & 2066, \text { Mohs, } \\ \text { Kohlensauren Baryt } & 1707 & 3962 & 2255, \text { Mohs. }\end{array}$

Die Grundform ist ein aus den Prismen 011 und 110 zusammengesetztes Rectangular-Octaëder.

Zu derselben krystallographischen Gruppe gehören bekanntlich auch das kohlensaure Blei, der kohlensaure Strontian und andere Salze, von denen ich einige hervorheben will.

Dic kohlensaure Magnesia bildet sich in hohen T'emperaturen inmer in der Form des Kalkspatbs. In viederen Temperaturen dagegen, wie G. Rose zuerst beobachtet hat, entstehen beide Formen: die des Kalkspaths sowrohl als die des Arragonits. Es ist daher wahrscheinlich, dals auch der Uebergang beider Formen bei ihr denselben Bedingungen unterworfen ist, wie bei dem koblensauren Kalk.

Das salpetersaure Ammoniak, Ȧm $\ddot{\ddot{\mathrm{H}}}$, zeigt unter dem Mikroskop in gewöholicher Temperatur nichts eigenthümliches. Es entsteht auf einer Glasplatie wie dic ineisten Salze in Dendriten, die von einem oder wenigen Punkten ausgehend sich über das ganze Feld verbreiten und sich von denen anderer Körper nur durch die Winkel zwischen den Armen und die Ausbildung der Enden mit Mühe unterscheiden lassen. Um die mikroskopischen Krystalle einzeln zu crlangen, muls man einen Weg einschlagen, welcher bei gröfseren Mengen gerade lie entgegevgesetzte Wirkung 
hervorbringen würdc. Man mufs nämlich den zum Theil noch flüssigen Tropfen stark durchrühren und dadurch die schon eutstandenen Krystalle zerbrechen. Viele der über die Glastafel zerstreutcn Fragmente ergänzen sich zu wohl ausgebildeten Krystallen von mannigfacher Lage gegen dic Axe des Mikroskops, und wenn dadurch auch nicht immer. die vollständige Bestimmung der Krystallform möglich wird, so wird doch wenigsteus die Utiterscheidung derselben von anderen Formen erleichtert.

Auf diese Weise entstehen im salpetersauren Ammoniak, je nach der Fläche, mit welcher der Krystall der Glasplatte aufliegt: Rectangel, Rlsomben mit gerade abgestumpften. Ecken und audere Tafeln, deren Haupt- und Randfächen auf cin Prisma des isoklinischen (ein- und cinnxigen) Systemes füliren.

Bei Anwendung ciner höheren Temperatur schmilzt und sublimirt das Salz. Der Schunelzpunkt ist etwa 145". Aber die cinmal gesclumolzcne Masse bleibt lange unterhalb dieser Temperatur flüssig, und das Sublimat ist oftmals auch dann flüssig, wenn die obere Platte diese Temperatur niemals erreicht hat. Das Salz erhält sich also nicht nur, sondern bildet sich auch in tropfbar flüssigem Zustande weit unterhalb scines Frierpunhtes, was nuch bei Phosphor, Schwefel und anderen Körpern hïulig beobachtet wird.

Diese Tropfen erstarren früher oder spïter zu Delldriten, oder wenn das Salz schon in Zersctzung begriffen war, zu einer sonar unter dem Mikroskop völlig amorph erscheinenden Gallerte, die sich jedoch zwischen den Nicol'schen Prismen ebenfalls als ein Aggregat von Kirystallen verhält, die zwar unregrelmälsig begränzt sind, deren optische Elasticitäts - Axen aber cbenso deutlich erscheinen wie in einem regelmä(sirg gebildeten Krystalle.

Aber wie die Krystalle des gewöbnlichen Salpeters erleiden auch die des salpetersauren Ammoniaks durch eine Erwärnuug, die hier $100^{\circ}$ nicht erreicht, cinc gänzliche Umwandlung, die man zwar vicht in gewöhulichen Lichte, aber sehr deutlich zwischen den Nicol's wahrniumt, wo

Poggendorfl's Ann. Bd. XCIII. 
jedes mikroskopische Prisma oder jeder Dendriten-Arm nicht mehr wie vor der Erwärnung ein Individuum ist, sondern in mehrere durch zuweilen sichtbare Quersprünge von einander getrennte Stucke getheilt ist.

Wenn man andererseits die in hoher Temperatur gebildeten Krystalle unter das Mikroskop bringt, wo sie allmälig erkalten, so kann man die entgegengesetzte Verwandlung deutlich beobachten und zwar am besten, wenn man den Krystall im Mikroskop so stellt, dafs er zwischen den Nicol's dunkel bleibt. Alsdann wird sich bei einer gewissen, nicht blofs von der Temperatur abhängigen Zeit der bisher dunhle Krystall plötzlich erhellen und in melirere krystallinisclue Stücke von abweichender Polarisation zerfallen.

Also geht das salpetersaure Ammoniak, wenn es in niederer Temperatur gebildet war, durch Errvïrmung, und wenu es in höherer gebildet war, durch Abkühlung in eine andere Krystallform über.

Das salpetersaure Ammoniak krystallisirt in niedriger Temperatur in der Form des gewöhulichen Salpeters. Die Form des in höheren Temperaturen krystallisirten Salzes habe ich noch nicht bestimmen können, da es nur in Dendriten oder dichten Krystallhaufen zu erlangen war. Das in grölserer Menge in Wasserbade krystallisirte Saiz bestand nach dem Erkalten aus kleinen Fascrn und flachen Prisnen, deren Querschuitt in Reflexions-Goniometer gemessen werden konnte. Es waren rhombische Prismen mit der vorherrschenden Abstumpfung der scharfen Seitenkanten; die Flächen des Prisma neigten sich zur Abstumpfung fast genau unter $126^{\circ}$, also ganz wie in Salpeter. Die End. llächeu kounten zwar nicht gemessen werden, aber dic Kante, welche sie init der Hauptnäche machten, stand perpendikular auf den Kanten des Prismas. Das Salz war also schon in die Form des gewöhnlichen Salpeters tibergegangen.

Die bei der Bildung des salpetersauren Kalis auch in der Kälte so bäufiog auftreteuden Rhomboëder habe ich nie- 
mals ain salpetersauren Ammoniak beobachten künnen. Bei der Uebereinstimmung aller übrigen Erscheinungen ist es jedoch sebr wahrscheinlich, dafs auch in deın salpetersauren Ammoniak die in hölerer Temperatur normale Form die rhomboëdrische sey.

Das Natron steht krystallographisch dem Kali weit ferner als das Ammouiak, und ist in dieser Beziehung dem Silberoxyde und auch dem Kalk näher verwandt als dem Kali.

Eine wirkliche Isomorphie des Natrons mit Kali, d. h. wo der eine Kürper deın anderen nicht etsva in einigen Procenten beigemischt ist, sondern wo er ihn in der Verbindung ganz ersetzt, findet sich aufserhalb des tesseraleu Systems nur in Feldspath und in cinem oder zwei künstlichen Salzen.

Haidinger beschreibt nämlich ein phosphorsaures Natron, das im monoklinischen Systeme krystallisirt und das logarithmische Grund-Verbältnifs

$$
9,8740 \quad 9,9143 ; 82^{\circ} 5^{\prime}
$$

hat, d. h. $\log B, \log C$ (wenn $A=1$ ) und der Winkel zwischen den Axen $B$ und $C$. Es hat nach Clark die Formel $\dot{\mathrm{N}} \mathbf{a}^{2} \dddot{\mathbf{P}} \mathbf{H}^{\text {!s }}$.

Ein von Mitscherlich gemessenes und analysirtes Salz hat auf dieselbe Weise berechnet

$$
9,8699 \quad 9,9143 ; \quad 82^{\circ} 55^{\prime}
$$

und die chemische Fornel $\dot{\mathrm{K}} \dot{\mathrm{Na}} \dddot{\mathrm{P}} \dot{H}^{\prime}$ ?. Auch die Ausbildung ist bei beiden Salzen übereinstimmend; sie sind also entschieden isomorph.

Die rationelle Formel des Mitscherlich'schen Salzes ist unstreitig $\dot{\mathrm{K}} \dot{\mathrm{Na}} \dot{\mathrm{H}} \ddot{\mathrm{P}}+16 \dot{\mathrm{H}}$, also in dem sogenaniten Krystallwasser ist zwei Mal soviel Sauerstoff als in dem Salze selbst. Clark's Salz würde seyn $\dot{\mathrm{Na}}{ }^{2} \dot{\mathrm{H}} \ddot{\mathrm{P}}+14 \dot{\mathrm{H}}$. Ist dieses richtig, so findet in der Zusammensetzung der Salze gar keine Uebereinstinmung statt. Aber der Wassergebalt wird oft selbst bei den besten Analysen unrichtig angegeben. Sollte sich bei einer genauen Analyse auch 
in diesem Salze $16 \dot{\text { fl }}$ ergeben, so wije die Basis der Salze

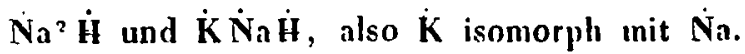

Sieht man ron dieser also noch zweifelhaften Isomorphie ab, so ist den Feldspath ausgenommen, wo auch noch Zweifel stattinden, dic cinzige zurerlässige lsomorphie von $K$ und $N a$ bei dem salpetersaurnn Salze, in dem

$$
\dot{K} \dddot{\mathrm{H}} \text { und } \dot{\mathrm{N}} \dddot{\dddot{H}} \text {, }
$$

beide in Rhounboëdern ron fast gleichen Dimensionen und Spaltungsrichtungen krystallisiren.

Iudessen labe ich das salpetersaure Natron bis jetzt blofs in rhomboëdrischen Formen gesehen. Eine bis zum Schmelzen gehende Erhitzung bringt keine Verïnderung in seiner Krystallform bervor.

Das saure kohlensaure Ammoniak, $\dot{A} m \dot{\mathrm{H}} \ddot{\mathrm{C}}^{2}$, hat nach G. Rose's Messungcu das logarithnische Grund-Verhiilinifs

$$
0,1720 \quad 0,3983 \quad 0,2260
$$

in isoklinischen System, wounit auch die Angaben ron Miller und Deville übereinstimmen. Es ist also dem kohlensauren Baryt beinahe gleicl. Nan gab ihm früher $\frac{1}{2}$ M.G. Wasser mehr, aber nach Deville's Untersuchungen ist es, wie ich schon in meinem Systeme der Krystalle S. 1.17 vermuthete, dem $\dot{K} \dot{H} \ddot{C}^{2}$ nnalog zusammengesetzt.

Die in gewöhnlicher Temperatur gebildeten Krystalle erleiden kcine Veränderung in ciner bis zum Verschwinden des Salzes steigenden Wärme. Aber zuweilen schiefst es in monoklinischen, von G. Rose gemessenen Formen an. Es ist also ebenfalls dimorph. Ich habe die Bedingungen des Ueberganges noch nicht untersucht; da das Salz in seiner Arragonit-ähulichen Form in höherer Temperatur normal ist, so gehört die monoklinischẹ Form vermuthlich der niedrigeren Temperatur an:

Auch das saure kohlensaure Kali, $\dot{\mathrm{K}} \dot{\mathrm{E}} \ddot{\mathrm{C}}^{2}$, ist dimorph. Seine gewöhnliche Krystallform, welche der des monoklinischen $\dot{A} m \dot{\mathrm{H}} \ddot{\mathrm{C}}^{2}$ gleich ist, verändert sich nicht, wemn das 
Salz bis zII seiner Zersetzung erlitzt wird. Aber in Moment seiner Eutstehung hat es noch eine 2 weite, bald zerstörte Form, die aber noch nicht genauer zu bestin. men war.

Die Isomorphie des $\dot{A} u \dot{H} \ddot{C}^{2}$ nit dem salpetersauren Ammoviak und dem Arragonit ist nicht ohne Interesse. Zieht man die Sauerstoff - Miscliungs - Gewichte zusammen, so sind die Formeln dieser drei isomorphen Salze, wenn man das $\dot{\mathrm{C} a} \ddot{\mathrm{C}}$ verdoppelt,

$$
\operatorname{Am} \mathrm{O}^{6} ; \mathrm{AmHC} \mathrm{C}^{2} \cdot \mathrm{O}^{6} ; \mathrm{CaCaC}^{2} \cdot \mathrm{O}^{6} \text {. }
$$

Ich will die Vermuthungen, welche sich an die Vertretung vou II $\mathrm{C}^{2}$ durch knüpfen, nicht weiter verfolgen. Was dic Vertretung vou 2 Ca durch AmH betrifft, so entlält sie nichts Unerwartetes. Aber man darf nicht vergessen, dafs so hiunlig auch HI wie cin Radical auftritt, dic Beispiele, wo II ein Radical oder überhaupt einen anderen Körper auch krystallographisch ersetzt, sehr sclten sind.

Folgendes sind dic einzigen mir bekannten Beispielc:

1) Der Ytterspath nach Berzelius und Schecrer's Analysen $=\dot{Y}^{\prime} \ddot{P}$, krystallisirt in eillem Quadrat-Octaëder, das einen Endhanten-Winhel von $121^{\circ}$ hat.

Der vou Mitscherlich analysirte und hrystallographisch bestimmle kila $\dddot{\mathbf{F}}$ hrystallisirt in cincm QuadratOctä̈der mit deu Endkanten-Winhel von $122^{\circ} 16^{\circ}$ also $\dot{\mathbf{Y}}^{3}=\dot{\mathrm{K}} \dot{\mathrm{H}}^{\prime}$.

2) Der Haidingerit nach Turner's Analyse $\mathrm{C}_{\mathrm{a}^{2}} \mathrm{As}_{\mathrm{H}} \mathrm{H}^{+}$, hat nach Il aidiuger's Messung das Grund-Verhïlnifs

$$
0,3051 \quad 0,3871 \quad 0,0820
$$

und als Grundlorm cin gerades rectanguliircs Prisma.

Das arsenihsanre Natron, Na As i $^{*}$, hat nach Milsclicr. lich das Grund-Verhiiltuils

$$
\text { 0,3051 0,3891 0,0578, }
$$

also sind beide, wenn nicht etwa die Spalungs Richlung!n cincn Unterschied macben, isomorpl. 
Die rationellen Formeln beider Salze sind

$$
\dot{\mathrm{Ca}}{ }^{2} \dot{\mathrm{H}} \dddot{\mathrm{As}} \dot{\mathrm{H}}^{3} \text { und } \dot{\mathrm{Na}} \dot{\mathrm{H}}^{2} \dddot{\mathrm{As}} \dot{\mathrm{H}}^{2}
$$

was, wenn T urner's Analyse richtig ist, unvereinbar wäre. Aber der Haidingerit wird, wie ich glaube, von einigen Chemikern $\dot{C} a^{2} \dot{H} \ddot{A s}^{2} \dot{H}^{2}$ gesetzt und dann wärc $\dot{C} a^{2}$ durch $\dot{\text { Na }} \dot{H}$ vertreten.

3) Der Dioptas, CüH $\ddot{\mathrm{Si}}$, und der Williamit, $\dot{\mathrm{Zn}}^{2} \ddot{\mathrm{Si}}$, krystallisiren in Rhomboëdern, jener von $126^{\circ} 17^{\prime}$ nach $\mathrm{Ph}$ il lips, dieser von $128^{\circ} 30^{\prime}$ nach Levy. Der Dioptas ist zwar hemiëdrisch, aber die bei dem Willianit vorkommenden Flüchen bleiben von dieser Hemiëdrie unberührt. Allein die Unterschiede in der Lage der Spaltungslächen und der Ausbildung machen die Isomorphie sehr unwahrscheinlich. Auch kann die Krystallform des Dioptases norh nicht als ganz bekannt angesehen werden, da mehrere der bei ilun angegebenen Flächen wahrscheinlich verschiedenen zwillingsartig verbundeneu Individuen angehören.

$\mathrm{Zu}$ diesen Beispielen tritt noch die Isomorphie des sauren koblensauren Ammoniaks mit dem kohlensauren Baryt oder Kalk, so dals bis jetzt höchstens drei Beispiele von Isomorphie des Wassers mit einer Basis bekannt sind ').

In der folgenden Tabelle habe ich die oben betrachteten kohlen - und salpetersauren Salze zusammengestellt. a ist die noch unbekanote Forın des saurcu kohlensauren Kalis, $\beta$ die monokliniscbe Form desselben Salzes, $\gamma$ und $\delta$ die Form des Arragonits und des Kalkspaths. Die Anord. nung ist unter der Voraussetzung getroffen, dals $\beta$ gegen $\alpha$, und $\gamma$ gegen $\beta$ in einem ăhnlichen Verhältuisse stehen, wie $\delta$ gegen $\gamma$; dafs also das fruber gestellte Zeicheu die ciner

1) Auch die Vertretung des Chlors durch Wasserstoff ist krystallugraphisclı bis jetzt nur in einem eipzigen Falle bestätigt, nämlich bei dęn von G. Rose gemessenen Isatin und Chlor-odes Brom-Isalid, die, wie ich beiläufig erwähnen will, dem Haidingerit ganz isomorph sind. $\mathrm{Zu}$ derselben Gattung gehört auch der Hopeït, der eio burax-oder phosphorsaures Salz zu seyn scheint, und das Anemonio nacls meinen Messungen. Mellithsaures Natron nach meiner Messung, und Myroxokarpin nach Miller stelsen ihm ebenfalls schr noth. 
niedrigeren Temperatur zukommende Krystallform eilles Salzes sey.

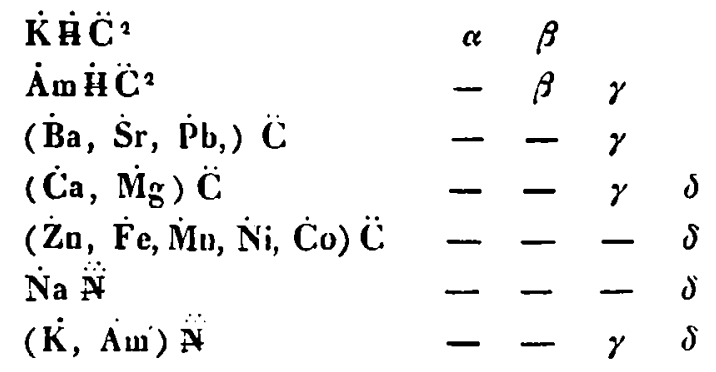

Man sieht, dafs das saure kohlensaure Kali, wclches krystallographisch gar nichts gemein bot unit kohlensaurem Zink, mit diesem durch das saure kohlensaure Ammoniak und den kohlensauren Kalk verbunden wird; sie gehören also trotz ihrer Verschiedenbeit einer krystallographischen Gruppe an.

Man kenut noch die Krystallform ciniger Körper, be denen man ihrer Zusammensetzung wach eine Isomorphie mit der oben bezeichneten Gruppe erwarten sollte.

Der Baryto-Calcit ist monoklinisch, aber verschieden voin sauren kohlensauren Kali.

Das salpetersaure Silber sollte, wie andere Silbersalze, dem Natron-Salze isomorph seyn. Es ist aber isoklinisch und zwar in ciner mit der des Arragonits nicht $2 u$ vereinigenden Fortn. Ich habe mich überzeugt, dafs es in einer bis zur Zersetzung steigenden Temperatur keine Veränderung seiner Forın erleidet.

Die salpetersauren Baryum-, Strontium- und Bleioxyde krystallisiren in Würfeln, Octaëdern und Pyritoëdern. Auch bei diesen drei Salzeu habe ich in hohen Temperaturen keine Veränderung der Form beobachtet.

Das salpetersaure Lilhion hat wenigsteus zwei Hydrate, von denen das eine schnell vorübergehend in regulären Octaëdern krystallisirt. Zu gleicher Zcit mit den gewöhnlichen Hydrat entsteht ein in höheren Temperaturen unver. ändertes Salz iı Würfelı und Octaëdern, deren Kanteu und 
Ecken in der Regel abgestumpft sind. Es erbält sich mit scharfen Kanten in einer durch Zerfliefsen der Hydrate cntstandenen Flüssigkeit. Es ist allem Anscheine nach wasserfrei. Ein zweites wasserfreies aber sehr zerfliefsliches salpetersaures Lithion entsteht durch Erwärmen des gewöhnlicheu Hydrates oder durch Krystallisation einer Lösung in hohen Temperaturen. Seine Formen künnen unter dem Mikroskope uiclit bestimunt werden, aber es polarisirt stark das Licht und ist daber von dem tesseralen Salze leicht zu unterscheiden.

Das salpetersaure Lithion ist also ebenfalls dimorph und es ist möglich, dals es in der einen, in der niedrigen Temperatur normalen Forn, mit dem salpetersauren Baryt isomorph ist.

Man wird mit der Zeit auch die Formen des BarytoCalcits, des salpetersauren Silbers und Baryts mit denen unserer Tabelle verbinden können und dadurch die Reihe der Krystallformen, welche ähnlich zusamuengesetzte aber krystallographisch verschiedene Körper mit einander verbindet, weiter ausdehnen.

Ich werde in einiger Zeit Golegenheit haben, ähuliche Reihen auch in anderen Kürper-Klassen nachzuweisen.

11I. Krystollochermische Monographie der Oxalsäure und ihrer Salze; con C. Rammelsberg.

\section{Erste Abtheiluag.}

Die oxalsauren Salze und Doppelsalze krýstallisiren zum Theil so schün, dafs cine Untersucbung ibrer Form von grofsem Interesse ist. $\mathbf{\Lambda}$ ufserdem ist auch manches Glied dieser Reihe noch nicht oder nur unvollstäudig bekannt. In Nachfolgreudeu habe ich mich bemülit, das bisher Bckanute zu revidireu, und die vorbandenen Lücken so viel als unöglich auszufüllen 